\title{
News from ASH 2018-how can we afford innovation in the future?
}

\author{
Dominik Wolf
}

The latest data presented at ASH 2018 (and also EHA 2019) document the continuous success in improving the outcome and tolerability of anti-cancer therapies and the management of coagulation disorders in hematology. Important topics are summarized in short reviews in this issue of MEMO [1-4]. Exemplarily, the integration of oral BTK and BCL-2 inhibitors into the first-line management of chronic lymphocytic leukemia (CLL) highlights the evolvement from classic chemo-immunotherapy to more targeted approaches using oral compounds guaranteeing better tolerability as well as higher efficacy in almost all CLL patient populations. Improved MRD negativity rates also suggest a marked improvement of long-term progression-free survival (PFS) and overall survival (OS) outcomes, even though the optimal sequencing of the currently available plethora of therapies in various diseases (most relevant in myeloma and CLL) still remains to be determined. In general, however, it is probably most promising to apply the most potent drugs or drug combinations (such as quadruplet induction in myeloma) to achieve the deepest response rates, thereby reducing the likelihood of subclonal disease evolution linked to increased aggressiveness of the disease in subsequent relapse. I was also impressed by additional data on cancer microenvironment/immune cell targeting achieved by BCMA-CD3 BiTEs in myeloma and CAR-T cells in

\section{Prof. Dr. med. D. Wolf $(\bowtie)$}

Internal Medicine V, Medical University of Innsbruck, Anichstraße 35, 6020 Innsbruck, Austria

Tyrolean Cancer Research Institute, Innrain 66, 6020 Innsbruck, Austria

Medical Clinic III, Oncology, Hematology,

Immuno-Oncology and Rheumatology, University Clinic

Bonn, Sigmund-Freud-Straße 25, 53127 Bonn, Germany

dominik.wolf@i-med.ac.at myeloma as well as various lymphatic diseases. These data exemplify how intelligent novel targeted treatment approaches can be applied, which are-even though larger and particularly randomized trial data are eagerly awaited-already showing dramatic improvement of the prognosis of relapsed/refractory lymphoma patients and considerable activity also in late-line myeloma treatment. It is tempting to speculate that more potent and fine-tunable CARTC technology platforms (e.g., SUPRA-CAR T cells), their earlier use and/or the combination of these targeted approached in intelligent sequential early-line treatment concepts or as add-on to classic targetedor chemo-therapies will further push PFS and OS in various hematological diseases. However, the most burning question even in high-income countries is the affordability of medical innovation, as often the mode of financing cutting-edge medical care is limited by financial restrictions even in many European countries. The pricing process of innovative therapies therefore has to be questioned, and innovation funds allowing for the rapid availability of novel, cost-intensive therapies for all of our patients have to be established. A more intense dialogue between manufacturers, regulators, payers, and patient associations is required to maintain the capacity of the academic sector to pursue relevant preclinical and clinical research in the field and to promote manageable reimbursement solutions. It is also our responsibility to make these innovative therapies available to patients all over the globe, which may require a more global perspective of cancer care.

Conflict of interest D. Wolf declares that he has no competing interests. 


\section{editorial}

\section{References}

1. Heintel D. Highlights on chronic lymphocytic leukemia presented at the 2018 American Society of Hematology Meeting. memo. 2019. https://doi.org/10.1007/s12254019-00521-y.

2. Panny M. ASH 2018-aggressive lymphoma. memo. 2019. https://doi.org/10.1007/s12254-019-0510-9.

3. Feistritzer C. Update in thrombosis and hemostasis: ASH meeting 2018. Memo. 2019. https://doi.org/10.1007/ s12254-019-0491-8.

4. Jeryczynski G, Krauth MT.ASH2018-Highlights in Multiple Myeloma. Memo. 2019. https://doi.org/10.1007/s12254019-0515-4.
Publisher's Note Springer Nature remains neutral with regard to jurisdictional claims in published maps and institutional affiliations.

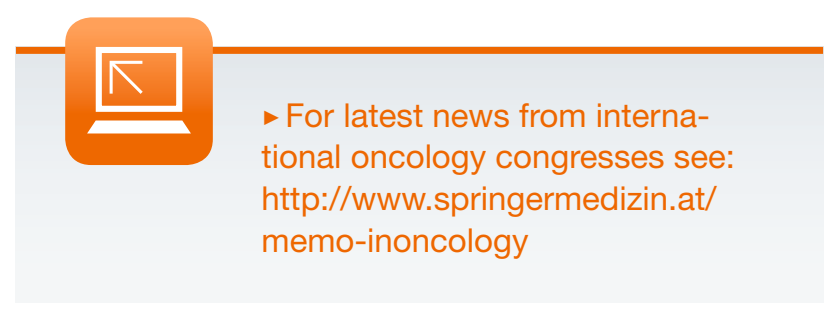

